\title{
INCIDÊNCIA DE SOROLOGIA POSITIVA PARA CITOMEGALOVÍRUS EM DOADORES DE TECIDO CUTÂNEO DO BANCO DE TECIDOS DO HOSPITAL DAS CLÍNICAS DA FACULDADE DE MEDICINA DA UNIVERSIDADE DE SÃO PAULO.
}

\author{
Incidence of Seropositivity for Cytomegalovirus in Skin Graft Donors at the Skin Bank of the \\ University of the São Paulo Medical School
}

Jader Joel Machado Junqueira', Aline Estefanes Eras', Eugênio Ferramundo Polo², Marisa Roma Herson² e Vera Aparecida dos Santos'.

\section{RESUMO}

Introdução: Considerando a provável relação entre a utilização de enxertos de pele infectados por citomegalovírus (CMV) e o desenvolvimento de doença grave por parte de receptor imunocomprometido, procurou-se analisar a incidência de anticorpos anti-CMV das classes IgG e IgM em doadores de tecido cutâneo do Banco de Tecidos do Hospital das Clínicas da Faculdade de Medicina da Universidade de São Paulo. Objetivo: Analisar a incidência de sorologia positiva para anticorpos anti-CMV das classes IgG e IgM em doadores de tecido cutâneo do Banco de Tecidos do HC FMUSP. Método: Realizamos um estudo retrospectivo com 112 resultados sorológicos obtidos no período de maio de 2001 a maio de 2006. Resultados: Nenhuma das amostras de sangue pesquisadas apresentou resultado positivo para pesquisa de anticorpos anti-CMV da classe IgM, enquanto a positividade para IgG foi de 91,07\% nas amostras pesquisadas. Assim, no grupo positivo para $\operatorname{IgG}$ (Grupo $1 \mathrm{n}=102$ ) o IgM foi negativo em 59 casos e não foi pesquisado em 43. Já no grupo negativo para IgG, (Grupo $2 \mathrm{n}=10$ ) o IgM foi negativo em 5 casos e não foi pesquisado em 5. Conclusão: Diante da alta prevalência de anticorpos anti-CMV da classe $\operatorname{IgG}$ em nossa casuística e da morbidade da doença citomegálica quando ativa em indivíduos imunocomprometidos, este trabalho sugere que os bancos de tecidos em geral e especificamente bancos de pele estabeleçam protocolos de triagem de tecidos que contemplem pesquisa sorológica para CMV.

Descritores: Transplante de Pele; Infecção por Citomegalovírus; Imunologia.

Instituição:

Departamento de Laboratório Central - Serviço de Imunologia do Hospital das Clínicas da Faculdade de Medicina da Universidade de São Paulo.

2 Banco de Tecidos do Hospital das Clínicas da Faculdade de Medicina da Universidade de São Paulo.

Correspondência:

Dra. Vera Aparecida dos Santos.

Serviço de Imunologia da Divisão de Laboratório Central do Hospital das Clínicas da Faculdade de Medicina da Universidade de São Paulo.

R. Dr. Enéas de Carvalho Aguiar, 155 - 2Andar / Bloco 5 - Cerqueira César

CEP: 05403-010 - São Paulo - SP

Tel.: 11 3069-6131

E-mail: vera@usp.com.br

Recebido em: 05.03.2007

Aceito em: 05.06.2007

\section{INTRODUÇÃO:}

Atualmente, tem-se verificado um aumento substancial das taxas de sobrevivência dos pacientes com queimaduras amplas (> 50\% da superfície corpórea), apesar das altas taxas de morbidade e mortalidade estarem ainda presentes. ${ }^{1}$ Nesses pacientes, a rápida cobertura da área cruenta resultante do trauma se faz necessária, restaurando as importantes funções da pele de regulação térmica, controle de perda de fluidos, além de ser uma barreira contra infecções. ${ }^{2}$ Nesse contexto, desde a década de 50 bancos de pele têm possibilitado o transplante de enxertos alógenos, consolidando seu papel como substitutos cutâneos temporários. Sua aplicação leva à diminuição da dor e da contaminação bacteriana comparado a curativos oclusivos. ${ }^{3,4}$

Os aloenxertos podem ser utilizados tanto "a fresco" (refrigerados), após criopreservação, ou ainda após processamento em altas concentrações de glicerol.

Inúmeros estudos epidemiológicos têm sugerido uma relação entre a utilização de aloenxertos com sorologia positiva para citomegalovírus (CMV) e o risco de desenvolver a infecção pelo vírus em pacientes queimados; tendo sido constatado que essa infecção contribui para o aumento da morbidade e mortalidade daqueles pacientes. ${ }^{5-7}$ Nesse fato reside a importância da avaliação da presença de sinais imunológicos da doença ativa ou contato dos doadores potenciais de tecidos cutâneos. 


\section{MÉTODO:}

Foram compilados em um estudo retrospectivo os resultados sorológicos obtidos com a pesquisa de anticorpos anti-CMV das classes IgM e IgG de doadores de pele do Banco de Tecidos do Hospital das Clinicas da Faculdade de Medicina da Universidade de São Paulo no período de maio de 2001 a maio de 2006.

Durante os cinco anos de análise, foi recebido um total de 148 sorologias de doadores de tecidos cutâneos (61 femininos, 87 masculinos), provenientes de cinco instituições da cidade de São Paulo: Instituto Dante Pazzanese - IDP (32), Hospital Israelita Albert Einstein - HIAE (3), Universidade Federal de São Paulo - UNIFESP (32), Hospital Central Irmandade da Santa Casa de Misericórdia de São Paulo - ISCMSP (13) e HC FMUSP (68).

A pesquisa sorológica foi feita no Serviço de Imunologia da Divisão de Laboratório Central através de método ELISA (Enzyme Linked Immunosorbent Assay). Nos três primeiros anos do estudo, o procedimento era realizado de forma automatizada com a utilização do aparelho AXSYM, marca ABBOTT ${ }^{\circledR}$. Há dois anos o procedimento tem sido realizado, ainda de forma automatizada, no aparelho Cobas Core, da marca Roche ${ }^{\circledR}$.

A pesquisa de anticorpos anti-CMV, principalmente os da classe IgM, nem sempre é procedimento de rotina em todos os hospitais na triagem de doadores de múltiplos órgãos. Assim, para efeito deste estudo, 36 doadores de tecidos cutâneos sem sorologia para CMV foram excluídos, devido à ausência da triagem sorológica completa (IgM e IgG). Foram incluídas amostras de apenas 112 doadores, sendo 68 do sexo masculino e 44 do sexo feminino com a idade média de 41,2 $\pm 12,8$ anos, variando de 11 a 65 anos. As amostras foram agrupadas de acordo com o resultado sorológico obtido na pesquisa de anticorpos anti-CMV das classes $\operatorname{IgM}$ e $\operatorname{IgG}$ em:

Grupo 1. IgG positivo e IgM negativo ou não pesquisado;

Grupo 2. IgG negativo e IgM negativo ou não pesquisado.

\section{RESULTADOS}

Com relação aos grupos, obtivemos os seguintes resultados (Tabela 1):

Tabela 1. Resultados das Sorologias para Anticorpos Anti-CMV

\begin{tabular}{|c|c|c|c|}
\hline \multicolumn{2}{|c|}{ IgG positivo $(91,07 \%)$} & \multicolumn{2}{|c|}{ IgG negativo (8,93\%) } \\
\hline IgM negativo & IgM não pesquisado & IgM negativo & IgM não pesquisado \\
\hline 59 & 43 & 5 & 5 \\
\hline
\end{tabular}

Grupo 1 ( $\operatorname{IgG}$ positivo e IgM negativo ou não pesquisado) - 102 doadores, $59 \mathrm{com} \operatorname{IgG}$ positivo e IgM negativo; $43 \mathrm{com}$ IgG positivo e IgM não pesquisado.

Grupo 2 (IgG negativo e IgM negativo ou não pesquisado) - 10 doadores, $5 \mathrm{com}$ IgG negativo e IgM negativo; 5 com IgG negativo e $\operatorname{IgM}$ não pesquisado.

Nenhuma das amostras de sangue pesquisadas apresentou resultado positivo para pesquisa de anticorpos anti-CMV da classe IgM. Obtivemos positividade de IgG em $91,07 \%$ das amostras e nenhum caso de IgM positivo.

\section{DISCUSSÃO}

As infecções por CMV são muito freqüentes, porém observa-se que a doença clínica é rara em crianças e adultos imunocompetentes. Entre adultos imunocompetentes, de $30 \%$ a $90 \%$ apresentam anticorpos IgG-CMV presentes no organismo, sendo descritos como soropositivos para CMV. ${ }^{8-10}$ Estudos de soroprevalência de anticorpos anti-CMV na população mundial demonstraram que o CMV ocorre em todas as regiões do mundo, sendo inversamente proporcional ao status socioeconômico do local. ${ }^{11,12}$

Em indivíduos imunocompetentes, a infecção pelo CMV não é capaz de expressar qualquer efeito patogênico, já que as células infectadas pelos vírus são destruídas por células $\mathrm{T}$ citotóxicas específicas e a infecção procede de forma assintomática. A presença dessa infecção assintomática é baseada na detecção de anticorpos anti-CMV nos fluidos corporais. O período de incubação é de quatro a 12 semanas, quando o antígeno pode ser detectado. Nesse período há o aparecimento de anticorpos anti-CMV da classe $\operatorname{IgM}$ e, mais tardiamente, aumento de cerca de $400 \%$ no nível de IgG-CMV. ${ }^{13}$

$\mathrm{O}$ vírus pode permanecer latente no interior de vários órgãos e ser reativado em decorrência de depressão da imunidade celular, como por exemplo, em situações como gravidez, AIDS, uso de drogas imunossupressoras ou grandes queimados. ${ }^{8,14-16}$ Alguns estudos sugerem que a imunossupressão pós-queimadura ${ }^{17}$, transfusão de sangue ${ }^{18}$ e o transplante de pele de doadores sorologicamente positivos para $\mathrm{CMV}^{19}$ podem ser considerados fatores de risco para o desenvolvimento de infecção por CMV. ${ }^{20}$

Nestes indivíduos imunocomprometidos a infecção latente do CMV transmitida através do transplante de tecidos pode tornarse sintomática, resultando em sérias complicações, devido à disseminação do vírus pelo organismo e possível envolvimento de órgãos. São descritas diversas manifestações clínicas, entre as quais a mais comum é a retinite, responsável por $85 \%$ dos casos de sintomas clínicos do CMV. Doenças gastrintestinais são a segunda manifestação mais comum, e incluem esofagite, colite, gastrite e hepatite. $\mathrm{O}$ acometimento do sistema nervoso central ocorre em menos de $1 \%$ dos $\operatorname{casos}^{12,14}$.

Estudos na Literatura mostram uma taxa de soroconversão (aparecimento de anticorpos anti-CMV) em pacientes anteriormente soronegativos que receberam aloenxertos de pele de cadáver soropositivos variando entre 17 e 22,7\% $\cdot^{5-7}$ Essa incidência é similar à de soroconversão em pacientes submetidos a transplante de órgãos sólidos, em que a importância da análise sorológica dos doadores já é bem aceita atualmente. ${ }^{21-23}$

Infecções recorrentes por CMV podem ser causadas por reativação do vírus causador da infecção primária ou por reinfecção. A reinfecção tem sido observada em casos de exposição a cepas diferentes de CMV., ${ }^{9,12-16}$

Apesar do mecanismo de transmissão do CMV ainda não ter sido determinado ${ }^{7}$, assim como o real potencial de transmissão viral pelos aloenxertos de pele, a análise sorológica de doadores de pele em bancos de tecido tem se mostrado cada vez mais importante, tendo em vista as possíveis repercussões clínicas envolvidas no caso da infecção por CMV em indivíduos imunocomprometidos. Além disso, pacientes com sorologia positiva para a infecção apresentam maior tempo de estadia hospitalar, comparados àqueles sem evidência sorológica ${ }^{5}$, o que acaba por aumentar os custos com o tratamento, e, principalmente, gerar maior risco de submeter esses pacientes a outras infecções. 
No Banco de Tecidos do Hospital das Clinicas de São Paulo - FMUSP, a triagem sorológica complementar para CMV préliberação dos tecidos é feita rotineiramente em amostras adicionais de sangue pela pesquisa de anticorpos das classes $\operatorname{IgG}$ e $\operatorname{IgM}$. Entretanto, essa ainda não é a rotina entre todos os bancos de tecidos ou grupos de transplante de órgãos que participam da rede. Significantemente, em nosso estudo foram excluídos 36 doadores (quatro captados na ISCMSP, dois no HIAE e 30 na UNIFESP) devido à ausência da triagem sorológica completa (IgM e IgG) considerada "irrelevante" para doadores de órgãos. Há ainda uma não uniformidade dos métodos de análise sorológica entre os hospitais, que variaram de acordo com a procedência do material, uma vez que cada instituição fornecedora utiliza o "kit comercial" que adquiriu através de seu próprio setor de compras, já que não existe uma central de compras organizada pelo Serviço Público.

\section{CONCLUSÃO:}

Diante da alta prevalência de anticorpos anti-CMV da classe IgG em nossa casuística e da morbidade da doença citomegálica quando ativa em indivíduos imunocomprometidos ${ }^{8,13-16}$, este trabalho sugere que os Bancos de Tecidos em geral e especificamente Bancos de Pele diante de sua clientela imunologicamente comprometida, estabeleçam protocolos de triagem de tecidos que contemplem necessariamente a pesquisa sorológica para CMV.

\section{ABSTRACT:}

Introduction: Considering the likely link between the use of cytomegalovirus (CMV) infected skin grafts and the development of severe disease in immunocompromised recipients, we analysed the incidence of IgM and IgG anti-CMV antibodies of skin graft donors of University of São Paulo Medical School Skin Bank. Purpose: To analyze the incidence of soropositive skin graft donors for IgM and IgG anti-CMV antibodies at the University of São Paulo Medical School Skin Bank. Method: We performed a retrospective study in 112 screening results from skin graft donors at the Skin Bank of the University of São Paulo Medical School from 2001 to 2006. Results: None of the serum samples presented IgM anti-CMV, while IgG anti-CMV was found in $91.07 \%$ in this screening. 102 positive donors for IgG anti-CMV, IgM anti-CMV were negative in 59 cases (58\%); in 43 cases (42\%) the IgG screening was not performed. 10 negative donors for IgG anti-CMV, IgM anti-CMV were negative in 5 cases $(50 \%)$, and in $5(50 \%)$ cases, the IgG screening was not performed as well. Conclusion: Considering the high IgG anti-CMV antibody rates in skin graft donors and the morbidity as acute disease in immunocompromised hosts, this study outlines the importance of a careful screening including CMV screening in donors as routine in tissue and especially in skin banks.

Keywords: Skin Transplantation; Cytomegalovirus Infection; Immunology.

\section{REFERÊNCIAS}

1. Wolf SE, Rose JK, Desai MH, e cols. Mortality determinants in massive pediatric burns: An analysis of 103 children with $>$ or $=80 \%$ TBSA burns $(>$ or $=70 \%$ fullthickness). Ann Surg. 1997;225(5):554-65.

2. Garrison JL, Thomas F, Cunningham P. Improved large burn therapy with reduced mortality following an associated septic challenge by early excision and skin allografting using donor-specific tolerance. Transplant Proc. 1995;27(1):1416-8.

3. Kobayashi H, Kobayashi M, McCauley R, e cols. Cadaveric skin allograft cytomegalovirus transmission in a mouse model of thermal injury. Clin Immunol. 1999;92(2):181-7.

4. Shelby J, Saffle JR, Kern ER. Transmission of cytomegalovirus infection in mice by skin graft. J Trauma 1988;28(2):203-6.

5. Bale JF Jr, Kealey GP, Massanari RM, e cols. The epidemiology of cytomegalovirus infection among patients with burns. Infect Control Hosp Epidemiol. 1990;11(1):17-22.

6. Kagan RJ, Naraqi S, Matsuda T, e cols. Herpes simplex vírus and cytomegalovirus infections in burned patients. J Trauma. 1985;25(1):40-5.

7. Kealey GP, Aguiar J, Lewis RW 2nd, e cols. Cadaver skin allografts and transmission of human cytomegalovirus to burn patients. J Am Coll Surg. 1996;182(3):201-5.

8. Mussi-Pinhata MM, Yamamoto AY. [Congenital and perinatal infections] J Pediatr (Rio J). 1999;75(Suppl 1):S15-30. Portuguese.

9. Griffiths P. Cytomegalovirus infection of the central nervous system. Herpes 2004;11(Suppl 2):95A-104A. Review.

10. Taylor GH. Cytomegalovirus. Am Fam Physician. 2003;67(3):519-24. Review.

11. Gold E, Nankervis GA. Cytomegalovirus. Viral infections of human: epidemiology and control. Plenum Press, New York, 3를 Ed. Capítulo 8. 169. 1991.
12. Veronesi R, Focaccia R. Tratado de Infectologia - Livro 1. Editora Ateneu, $2^{\mathrm{a}}$ Edição. 190-196;150-5.

13. Anaizy N. Citomegalovírus in solid organ transplantation. Disponível em: http:// www.thedrugmonitor.com/cmv-1.html. Acessado em 31/10/2006.

14. Mandell GL, Douglas RG, Bennett JE. Principles and Practice of Infectious Diseases. Editora Churchill Livingstone, 3ㄹ Edição. 1159-1169.

15. Alford CA, Stagno S, Pass RF e cols. Congenital and perinatal cytomegalovirus infections. Rev Infect Dis. 1990;12(Suppl 7):S745-S753. Review

16. Brown HL, Abernathy MP. Cytomegalovirus infection. Semin Perinatol 1998;22(4):260-6.

17. Polk HC Jr, George CD, Wellhausen SR, e cols. A systematic study of host defense processes in badly injured patients. Ann Surg. 1986;204(3):282-99.

18. Preiksaitis JK, Brown L, McKenzie M. The risk of cytomegalovirus infection in seronegative transfusion recipients not receiving exogenous immunosuppression. J Infect Dis. 1988;157(3):523-9.

19. Shelby J, Shanley J. Transfer of murine cytomegalovirus by syngeneic skin grafts. Transplantation. 1987;44(2):318-20

20. Bale JF Jr, Kealey GP, Ebelhack CL, e cols. Cytomegalovirus infection in a cyclosporine-treated burn patient: case report. J Trauma. 1992;32(2):263-7.

21. Breinig MK, Zitelli B, Starzl TE, e cols. Epstein-Barr virus, cytomegalovirus and other viral infections in children after liver transplantation. J Infect Dis. 1987;156(2):273-9.

22. Merigan TC, Renlund DG, Keay S, e cols. A controlled trial of ganciclovir to prevent cytomegalovirus disease after heart transplantation. N Engl J Med. 1992;326(18):1182-6.

23. Goodrich JM, Mori M, Gleaves CA, e cols. Early treatment with ganciclovir to prevent cytomegalovirus disease after allogeneic bone marrow transplantation. $\mathrm{N}$ Engl J Med. 1991; 325(23):1601-7. 